\title{
BMJ Open Efficacy of a strategy-based intervention on text-level reading comprehension in persons with aphasia: a study protocol for a repeated measures study
}

Sarah-Maria Thumbeck, ${ }^{1}$ Philipp Schmid, ${ }^{2}$ Sophie Chesneau, ${ }^{3}$ Frank Domahs (D) ${ }^{1}$

To cite: Thumbeck S-M, Schmid P, Chesneau S, et al. Efficacy of a strategy-based intervention on text-level reading comprehension in persons with aphasia: a study protocol for a repeated measures study. BMJ Open 2021;11:e048126. doi:10.1136/ bmjopen-2020-048126

- Prepublication history and additional supplemental material for this paper are available online. To view these files, please visit the journal online (http://dx.doi.org/10.1136/ bmjopen-2020-048126)

Received 22 December 2020 Accepted 01 July 2021

\section{Check for updates}

(C) Author(s) (or their employer(s)) 2021. Re-use permitted under CC BY-NC. No commercial re-use. See rights and permissions. Published by BMJ.

${ }^{1}$ Linguistics, University of Erfurt, Erfurt, Germany

${ }^{2}$ Psychology, University of Erfurt, Erfurt, Germany

${ }^{3}$ Orthophonie, Université du Québec à Trois-Rivières, TroisRivieres, Quebec, Canada

Correspondence to

Dr Frank Domahs;

frank.domahs@uni-erfurt.de

\section{ABSTRACT}

Introduction At least $68 \%$ of persons with aphasia (PWA) experience reading difficulties. Even though strategy-based interventions are a promising treatment approach for text level reading comprehension deficits in PWA, empirical evidence for their efficacy remains rare. The primary objective of this study is the analysis of the efficacy of a strategy-based intervention on text-level reading comprehension and on reading activities in PWA. Methods and analysis In a repeated measures trial, 24 PWA will first participate in a waiting period and then in a strategy-based intervention (14 face-to-face-sessions, 60 min each). We will apply two combinations of strategies to treat either the microstructure or the macrostructure, respectively. Participants will be randomly allocated to two parallel groups that will receive these combinations in interchanged sequences. Assessments will be implemented before and after each period as well as 3 and 6 months after the intervention. The primary outcome measure is text-level reading comprehension measured with a German version of the Test de Compréhension de Textes (TCT-D) and represented by the score TCT-D Total . A non-blinded and a blinded rater will evaluate the primary outcome measure. Secondary outcome measures will address specific reading functions, reading activities and cognitive functions. The sample size was determined with an a priori power analysis. For statistical analysis, we will use contrast analyses within repeated measures analysis of variance models. We expect significant improvements in primary and secondary outcome measures during the intervention as compared with changes during the waiting period.

Ethics and dissemination This study was approved by the ethics committee of Deutscher Bundesverband für akademische Sprachtherapie und Logopädie (20-10074-KA-MunmErw+Ko). Results and relevant data will be disseminated in peer-reviewed journals, at conferences and on the Open Science Framework.

Trial registration number DRKS00021411 (see Supplementary Table 1).

\section{INTRODUCTION}

Background and rationale

Acquired brain injury can impair reading from grapheme or word levels to the comprehension of written texts in the context of

\section{Strengths and limitations of this study}

- This study will support clinical decision making in aphasia therapy since it is the first repeated measures group study that evaluates effects of strategy-based interventions on text level reading comprehension.

- Based on theoretical models of text comprehension, the effects of two different combinations of strategybased interventions on microstructure and on macrostructure will be explored.

- Outcome measures do not only include reading functions and cognitive functions but also reading activities.

- Blinding of speech and language pathologists and patients will not be possible due to the nature of speech and language interventions but we will perform a blind duplication of the evaluation of the primary outcome.

- The effect size of the a priori power analysis was estimated based on more general treatment approaches in persons with aphasia due to the novelty of the specific investigated treatment approach.

cognitive communication disorders or aphasia. At least $68 \%$ of persons with aphasia (PWA) experience reading difficulties. ${ }^{1}$ With regard to more complex reading functions, prevalence may be even higher. ${ }^{2}$ Text-level reading difficulties constrain activities such as reading medication schedules, text messages, books, job requirement or contracts, which in turn restricts participation in life domains like communication, self-care, family roles, employment as well as domestic and social life. ${ }^{3-5}$ Despite these severe consequences, reading impairments following stroke are often treated insufficiently or remain untreated. ${ }^{6}$ Systematic reviews of Purdy et $a l^{7}$ and Watter et al indicate that the evidence for the efficacy of text-level reading comprehension treatments in PWA is limited. They suggest further investigation with more rigorous study designs as well as research on 
the contribution of cognitive and linguistic factors to text comprehension impairments in PWA.

Current cognitive theories suggest that reading is a constructive process based on an interaction between reader and text. ${ }^{910}$ Reading comprehension processes depend on visual processes, the conversion of visual input into a linguistic representation, general knowledge, and a reader's mental lexicon as well as orthographic and linguistic systems. ${ }^{11}$ When reading a text, readers form three levels of mental representation: The surface level, the textbase and the situational model. While the phrases 'funny girl' and 'the girl is funny' differ in their superficial appearance of exact words and phrases (the surface level), they convey the same semantic content. The semantic content of a text is represented in the textbase in propositional form, thus in an idea-level format. ${ }^{9} 12$ Propositions are "linguistic units consisting of a relational term (or predicate) and one or more arguments', ${ }^{12}$ for example, (FUNNY, GIRL) or (EAT, GIRL, CAKE), and allow to work with the semantic content of a text regardless of the exact wording. In a text, every proposition is linked to at least one other proposition by a relational term or a shared argument, which establishes local coherence. ${ }^{13}$ Following the Construction-Integration-Model by Kintsch, ${ }^{12}$ the textbase consists of microstructure (the whole "network of propositions that represent the meaning of a text', ${ }^{12}$ that is, all detailed information of a text) and macrostructure (the most relevant propositions, the gist).${ }^{12}$ Although the process of generation is unclear, the resulting macrostructure indicates that microstructure is reduced according to specific macrorules: selection (selection or deletion of (ir)relevant propositions), generalisation (replacing subordinate by superordinate propositions) and construction (general proposition instead of a sequence of propositions). Due to spreading activation in the propositional network, several plausible meanings may be constructed in the textbase. The activation values of incorrect elements decrease with richer context, and they will be suppressed in the integration phase. ${ }^{12}$ At the third level, the information from the textbase, the context and the reader's pre-existing knowledge are integrated continuously taking into account cognitive schemata like scripts (eg, processes) and frames (eg, situations, objects, institutions or character types). ${ }^{9}$ Thus, the meaning of a text is constructed and represented in individual coherent situational models in the episodic memory. Situational models can be considered as a form of inference. ${ }^{12}$ Inferences are cognitive processes or results that provide information not explicitly stated in a text. ${ }^{14}$ They may involve the automatic or controlled generation of new information, or the retrieval of preexisting knowledge from long-term memory to bridge gaps. ${ }^{12}$

Thus, text comprehension depends on a complex interaction of linguistic and cognitive resources. In PWA, on the one hand, cognitive resources such as verbal working memory, episodic memory, monitoring and cognitive flexibility may be impaired. ${ }^{15} 16$ On the other hand, receptive and productive language skills can be affected to various degrees. ${ }^{16}{ }^{17}$ Even though general aphasia tests (which often assess phonology, morphology or syntax) may detect only minor or no difficulties, PWA may encounter substantial problems in text comprehension, including a reduced reading speed. ${ }^{15} 18$ Moreover, incomplete lexical and syntactic information may affect the construction of the propositional textbase, especially the microstructure. ${ }^{15} 19$ Furthermore, local and global inferencing skills as well as the construction of macrostructures and of situational models may be impaired. ${ }^{1516}$ This may be explained by anomalous macrostructural processes or-since knowledge about the world is usually better preserved than linguistic components in PWA-as a consequence of impaired lexical and syntactic processing. ${ }^{19}$

To treat reading comprehension in PWA, oral, strategybased, cognitive and hierarchical reading treatments can be distinguished. ${ }^{7}$ In oral reading treatments, PWA read texts repeatedly aloud, either independently or together with a speech and language pathologist (SLP), to facilitate whole-word recognition and top-down processing (ie, using prior knowledge to enhance understanding) ${ }^{78}$ This may be particularly helpful to improve reading comprehension in severe aphasia. ${ }^{20}$ Hierarchical reading treatments ${ }^{21} 22$ were provided with systematic computerised visual-matching and reading comprehension tasks. Stimuli are available in English language and consisted of symbols, letters, numbers, words, phrases and sentences, whereas text level reading comprehension was neither included in stimuli nor in outcome measures. ${ }^{21}{ }^{22}$ Cognitive treatments focus on underlying cognitive functions and were conducted with Attention Process Training II/III (computerised exercises to train specific domains of attention $)^{23-25}$ or sequenced exercises addressing working memory (sentence grammaticality judgement and semantic categorisation of words across several sentences). ${ }^{26}$ They may be particularly beneficial for persons with mild aphasia. ${ }^{7}$ In strategy-based treatments, reading strategies are used to facilitate reading comprehension. ${ }^{8}$ Reading strategies are specific, targetoriented cognitive techniques applied either automatically or consciously and flexibly. They support linguistic and cognitive processes such as the maintenance of text contents and the construction of the textbase and the situational model. ${ }^{97}$ Watter et $a l^{8}$ distinguish visual strategies (eg, underlining, highlighting), content strategies (eg, identification of key words, summarisation, preview of headings) and (meta)cognitive strategies (eg, rereading, recall, self monitoring).

To date, the evidence base is limited: cognitive and strategy-based interventions have been explored in (multiple) case studies, oral and hierarchical reading treatments in randomised controlled trials (RCTs). A gold standard cannot be concluded. ${ }^{78}$

This study focusses on strategy-based treatments in PWA. With regard to PWA, reading strategies may be a suitable option to take into account both linguistic and cognitive processes. Strong evidence exists already 
for other populations such as children with reading or learning disorders.eg, ${ }^{28}{ }^{29}$ Moreover, strategy-based interventions are cost-effective due to their relatively low dosage. ${ }^{8}$ In contrast to other approaches, reading strategies are not confined to a predefined software or to specific languages, items or text types. They permit the lecture of authentic texts that are age appropriate, relevant and interesting for individuals, which may raise the motivation to engage in therapy. Despite these potentials of strategy-based interventions and despite PWA's ability to apply reading strategies ${ }^{30}$ the latest systematic review by Purdy $e t a l^{7}$ identified only four case studies ${ }^{31-34}$ that evaluated strategy-based treatments with a total of six PWA. Four of the patients improved their reading comprehension. ${ }^{7}$ Furthermore, Rogalski and Edmonds ${ }^{35}$ and Rogalski $e t a l^{36}$ found that two out of three patients improved in text-related measurements with Attentive Reading and Constrained Summarisation which contains the strategy of summarisation.

The strategies that were applied in the studies listed above are provided in online supplemental table 2. In these studies, mainly content strategies (identifying/ writing down key words and key messages, summarising paragraphs and chapters, creating mind-maps and using advance organisers) as well as a few visual strategies (highlighting key words, using cards to block lines above and below focused lines) and the cognitive strategy (re)reading sections aloud and silently were explored. Furthermore, SLPs implemented strategies that can only be applied with a second person, such as discussions to increase motivation, reading/writing aloud to the patient, shared reading and writing, foreshadowing, modelling of reading and writing, providing metaliteracy comments and providing questions about the text. ${ }^{31-36}$

Elements that require either a second person or textspecific additional material contradict autonomy and may not be useful in authentic reading situations. The strategies explored in PWA to date have predominantly focused on the macrostructure and on the situational model as well as on the integration of background knowledge and (meta)cognitive abilities. They have not been explored in group studies with PWA yet. Considering that the microstructure is particularly prone to impairment in PWA, these strategies could be supplemented by strategies that target the microstructure, that could be used without a second person or additional text-specific material, and that have been suggested for other populations. Strategybased programmes constructed for other populations include SQ3R (survey, question, read, recite, review), PQRST (preview, question, read, summarise, test) and reciprocal teaching (prediction, clarification, summarisation, question generation). ${ }^{10} 37$ Based on their metaanalysis, Mayer and Marks ${ }^{28}$ developed a strategy-based concept for children that includes the activation of prior knowledge, mental imagery, comprehension monitoring, summarising and asking questions. In students with learning disabilities, Souvignier and Antoniou ${ }^{29}$ found highest effects for the strategies activation of background knowledge, summarisation, monitoring, asking questions, identification of the topic as well as strategies focussing on the text structure. Schmidt ${ }^{38}$ suggests the use of referential links as a reading strategy. It is important that strategy combinations are more effective than the use of only one strategy. ${ }^{28}$ In view of these suggestions, we think that asking questions and using referential links could be implemented in ways that facilitate understanding of the microstructure.

Based on this preliminary evidence and on specific impairments in PWA in linguistic as well as cognitive resources, we will combine specific reading strategies that have been investigated in PWA or that have been suggested for other populations. We will group the strategies in (1) macrostructure and situational model and (2) microstructure and surface structure. To raise motivation and to take advantage of possibly unimpaired schemata, we will embed the strategies in product-oriented goals. ${ }^{39}$

\section{Objectives and research hypotheses}

The primary objective of this study is to determine whether the effects of a systematic strategy-based intervention on text-level reading comprehension and on activities and attitudes related to reading in PWA are superior to spontaneous recovery.

Secondary objectives are (1) to compare the effects of two types of strategy-based interventions on the comprehension of the microstructure and the macrostructure; (2) to investigate the relationship between microstructure, macrostructure and the situational model on the one hand and specific cognitive functions such as working memory, episodic memory, executive functions and selective attention on the other hand.

We expect the superiority of the intervention compared with the waiting period with regard to improvements in text-level reading comprehension and in activities and attitudes related to reading. Furthermore, we anticipate specific effects on the microstructure or the macrostructure depending on the strategies used.

\section{METHODS AND ANALYSIS}

The study protocol is based on the Standard Protocol Items: Recommendations for Interventional Trials 2013 statement. ${ }^{40}$

\section{Trial design}

The trial is designed as a repeated measures study in a superiority framework with a primary endpoint of textlevel reading comprehension. The effects of a strategybased intervention and of a waiting period will be compared in a single group design. The strategy-based intervention will consist of two elements (Intervention Micro and Intervention Macro). To explore strategyspecific effects and to control for order and sequence effects, participants will be allocated to two parallel groups who will participate in these two elements in counterbalanced order after the waiting period (see figure 1). 


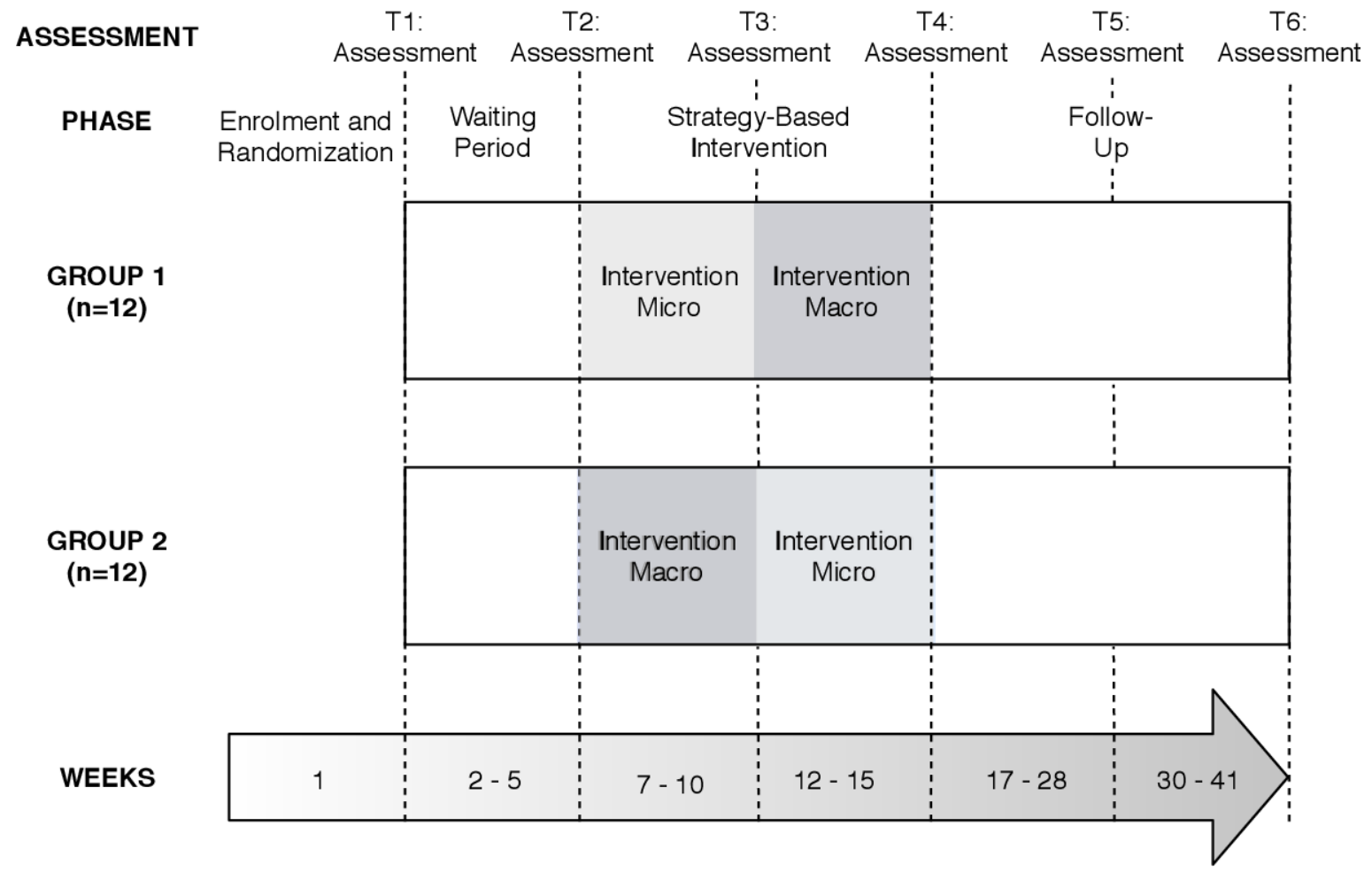

Figure 1 Trial design.

\section{Study setting}

Participants will be recruited in Germany (regional focus on urban and rural areas of Munich and Erfurt). Assessment and intervention sessions will be implemented in their regular rehabilitation centres, at the University of Erfurt, at the Ludwig Maximilian University of Munich or at participants' homes.

\section{Participants}

We will include persons with mild to moderate aphasia. For recruitment, we will advertise the study in newsletters of professional associations, send a flyer to rehabilitation centres, and contact aphasia support groups at meetings, by phone and e-mail. Table 1 shows the eligibility criteria.

\section{Sample size}

A priori power analysis using $\mathrm{G}^{*}$ Power $\mathrm{V} .3 .1^{41}$ revealed a minimum sample size of $\mathrm{N}=21$ to detect an effect size of $d=0.58$ with a power of .80 in a repeated-measures analysis of variance (ANOVA) ( $\alpha=0.05$; two measurements: eg, $\mathrm{T} 2$, T4; correlation among repeated measures $=0.60$ ). The effect size of the power analysis was informed by the effect size of previous research on speech and language interventions for PWA. ${ }^{42}$ We expect a drop-out-rate of $10 \%$ $(n=3)$. Thus, we will recruit a total sample size of $\mathrm{N}=24$.

\section{Assignment of interventions}

Participants will be allocated to two groups with the ratio 1:1 by stratified permuted block randomisation. With

\begin{tabular}{|c|c|}
\hline $\begin{array}{l}\text { Inclusion } \\
\text { criteria }\end{array}$ & $\begin{array}{l}\text { Age of at least } 18 \text { years } \\
\text { Aphasia according to Aachener Aphasie Test (AAT) }{ }^{45} \text { or no current symptoms/only residual aphasia according } \\
\text { to the latest AAT assessment but previously diagnosed aphasia and current language difficulties (subjective o } \\
\text { perceived by an SLP) } \\
\text { Anomalous scores in the German version of Test de Compréhension de Textes }{ }^{13} \text { (TCT-D) } \\
\text { Native language: German } \\
\text { At least } 3 \text { months postonset }\end{array}$ \\
\hline $\begin{array}{l}\text { Exclusion } \\
\text { criteria }\end{array}$ & $\begin{array}{l}\text { Global aphasia and/or severe problems in word level reading (score }<12 \text { in AAT subtest 'single word reading } \\
\text { comprehension') and/or severe problems in written language (score }<22 \text { in category 'written language' in } A A T \\
\text { Neurological, psychiatric or any other disease that impedes a (repeated) assessment and valid interpretation } \\
\text { with the AAT or TCT-D (particularly if the disease can result in decreasing or strongly fluctuating linguistic or } \\
\text { cognitive performance) } \\
\text { Premorbid dyslexia }\end{array}$ \\
\hline
\end{tabular}

SLP, speech and language pathologist. 
regard to prognostic factors, ${ }^{17} 4344$ we will stratify for age, severity of aphasia (age-adjusted score in the subtest Token Test of Aachener Aphasie Test ${ }^{45}$ ) and the TCT-D Total score. Randomisation and allocation will be implemented independently by Dr Thomas Lauer, scientific managing director of the ErfurtLab at the University of Erfurt, Germany. SLPs will enter enrolled participants' identification numbers along with their stratification factors into a table that Dr Thomas Lauer will use to generate blocks and to randomly allocate the participants to one of the two groups. The block sizes will not be disclosed, to ensure concealment.

\section{Intervention procedures and material}

The interventions will start in the beginning of 2021 . They will be implemented by ST and by qualified SLPs who work in a setting licensed by the German health insurance system. Between $\mathrm{T} 1$ and T4, the participants will not receive any other aphasia treatment.

Strategy-based intervention. The intervention period consists of two elements, Intervention Micro and Intervention Macro (see table 2).

To promote transfer, we will only use strategies that participants may apply without a second person, and we will inform participants about the possible use of reading strategies in everyday reading activities. Intervention Micro and Intervention Macro will each consist of seven 60 min sessions, provided twice a week in individual therapy. Additionally, the participants will apply the same strategies as homework for $60 \mathrm{~min}$ after each intervention session. Homework will be monitored with a timetable. During the intervention sessions, SLPs write down the answers of the participants.

All external SLPs who deliver the treatment will participate in a preparatory workshop. They will be provided with

- A manual that contains instructions, examples, structured guidance for facilitation and worksheets.

- Stimulus material: articles from magazines with increasing difficulty (based on readability index, word count, number of pictures) in a predefined randomised order for each participant.

Procedures of allocated interventions may not be modified. Whenever a participant cannot solve a given task, hierarchical cues will be offered:

1. Authentic situations, for example, 'If you tell your partner later on what that paragraph was about, which key word would you say?'

2. Semantic cues, for example, 'You said 'A hiker marched towards...'. Now we have to replace 'his house'. So, we are looking for another building. Which building does the hiker want to go to?'

3. Phonological cues, for example, 'I am thinking about a building that starts with c.'

Waiting period. The participants will not receive any form of aphasia treatment during 4 weeks prior to the intervention.

\section{Outcomes}

The outcome measures (see table 3) were chosen with regard to the levels functions and activities of The International Classification of Functioning, Disability and Health. ${ }^{5}$ Assessments will take place at six points (T1, T2, T3, T4, T5, T6) with T6 being optional (see figure 1). Instructions will be standardised without explicit encouragement to use reading strategies postintervention.

The primary outcome is text level reading comprehension defined as the score TCT-D Total measured with TCT-D. TCT-D allows to analyse specific text-level reading functions that may be impaired in PWA. TCT-D Total is the sum of microstructure and macrostructure scores across all subtests of TCT-D.

As secondary outcomes, we will collect scores of microstructure, macrostructure, the situational model and reading time with TCT-D. Reading activities as well as attitudes towards reading will be measured with an adapted German version of the Comprehensive Assessment of Reading in Aphasia (CARA) Reading Questionnaire $^{2}$ which was developed specifically for the needs of PWA. At T2 and T4, we will measure inferences and macrostructure using the MAKRO-Screening ${ }^{46}$ and verbal episodic memory using Wechsler Memory Scale IV (WMS IV) ${ }^{47}$ (logical memory 1). Cognitive functions that have been shown to be relevant for reading in PWA will be measured with subtests of the German versions of the WMS-Revised ${ }^{48}$ (digit span forward and backward), Farbe-Wort-Interferenz-Test ${ }^{49}$ and a subtest on attention of Aphasie-Check-Liste. ${ }^{50}$ We will analyse change in individual scores across the whole group and across the subgroups between specific assessment points. We will explore individual effects on functions, activities and participation as well as the potential generalisation of strategy use to everyday reading materials with a semistructured interview at $\mathrm{T} 4$.

\section{Blinding}

Participants will be blinded with regard to the specific hypotheses of the study. Due to the nature of behavioural speech and language therapy, neither participants nor SLPs can be blinded with regard to the intervention they receive or provide. Analysis of the main outcome measure TCT-D will be duplicated by blinded assessors for T1, T2 and $\mathrm{T} 4$ based on audio recordings and the level of agreement will be reported in the final trial report. ${ }^{5152}$

\section{Data collection and participant retention}

For all participants, the same data collection methods will be used. Data collection will be performed by ST and by participating SLPs based on manuals and protocol booklets to ensure standardised procedures. We will collect the data described in table 3. Points of assessment are provided in table 4 . For additional information on the assessment tools, see online supplemental table 3 .

Participants may withdraw from the study at any time. Participants that drop out after enrolment will be asked to participate in the TCT-D and in the CARA questionnaire 
Table 2 Strategies, procedures, rationales and examples; items in italics illustrate answers produced by the participants Intervention Macro: Macrostructure and situational model

$\begin{array}{ll}\text { Strategy } 1 & \text { Use of advance organisers and key words to create a mind map before and while reading } \\ \text { Product } & \text { Mind map } \\ \text { Procedures } & \text { 1. PWA finds out the topic of the text by using advance organisers such as title, pictures or } \\ \text { subtitles from the text }\end{array}$

Rationale

The activation of background knowledge is expected to facilitate the integration of the reader's knowledge and the new information from the text (and therefore the construction of the situational model). ${ }^{28}$ Advance organisers such as pictures can support paragraph level reading in PWA. ${ }^{56}$ Inferring a maximum of three key words about each paragraph will require to reduce microstructure to the most relevant information.

\begin{tabular}{|c|c|}
\hline Strategy 2 & Summarisation and rereading \\
\hline Product & News in short \\
\hline Procedures & $\begin{array}{l}\text { 1. PWA reads paragraph aloud } \\
\text { 2. PWA rereads } 2-3 \text { sentences of that paragraph aloud } \\
\text { 3. PWA rereads the same } 2-3 \text { sentences silently } \\
\text { 4. Text is covered, PWA summarises these sentences from memory based on macrorules }{ }^{12} \\
\text { and with specific restrictions (no personal opinion, no unspecific words) similar to Rogalski } \\
\text { and Edmonds }{ }^{35} \text { and Rogalski } e t a^{36} \\
\text { 5. Same procedure for the next } 2-3 \text { sentences from the same paragraph, until paragraph is } \\
\text { completed } \\
\text { 6. From memory, PWA adds } 1-3 \text { key words about the paragraph into the mind map } \\
\text { 7. Repeat from step one with the next paragraph }\end{array}$ \\
\hline
\end{tabular}

Rationale

We intend to facilitate the comprehension of the macrostructure and the construction of the situational model by actively applying the macrorules. We combine strategies previously described for PWA (use of keywords, writing down key messages, summarisation and rereading text). We will use a modified version of $A_{R C S^{35}}^{36}$ in order to integrate the cognitive strategy of rereading text aloud and silently and to target cognitive functions such as attention and maintaining information.

Example The young guy who wore a suit opened the door. He looked around, sat down and asked for the menu. He ate soup, lasagna and tiramisu, paid, and left.

$\rightarrow$ The guy ate a menu in a restaurant.

Intervention Micro: Microstructure and surface structure

$\begin{array}{ll}\text { Strategy } 1 & \text { Asking questions and providing answers } \\ \text { Product } & \text { Quiz } \\ \text { Procedures } & \text { 1. PWA skims a paragraph } \\ \text { 2. PWA underlines any part of the first sentence } & \text { 3. PWA asks for the underlined part of the sentence } \\ \text { 4. PWA generates (a) a synonym/paraphrase, (b) a semantically related answer, (c) logically } \\ \text { possible but wrong answer according to the text } \\ \text { 5. Repeat steps } 2-4 \text { until paragraph is completed, then repeat from step 1 } \\ \text { To date, this reading strategy has not been investigated in PWA apart from providing } \\ \text { predetermined questions. }{ }^{\text {7 }} \text { This procedure will allow PWA to ask questions on subordinate } \\ \text { propositions, which may facilitate the comprehension of the microstructure. Rearranging } \\ \text { sentence structures from statements into questions will only be possible with understanding } \\ \text { the links between the clauses. To ensure that participants will not stick to the surface structure } \\ \text { but access the semantic content of the answers to the questions, they will generate (a) a } \\ \text { synonym/paraphrase and (b) a semantically related answer. To address previously described } \\ \text { problems in cognitive flexibility in PWA and to integrate background knowledge, they will } \\ \text { generate c) a possible but incorrect answer to the question. }\end{array}$




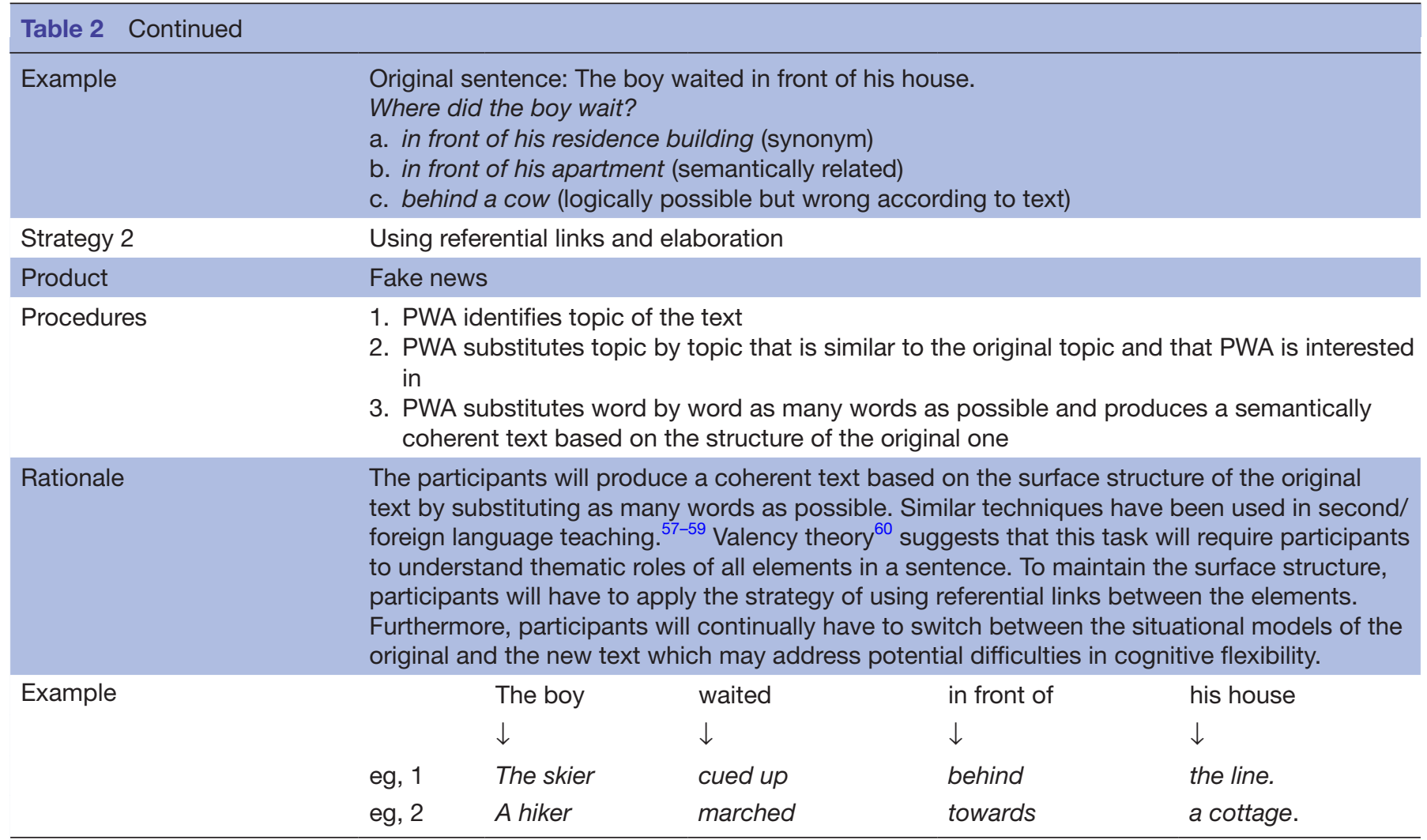

ARCS, Attentive Reading and Constrained Summarisation; PWA, persons with aphasia.

for a final assessment. They will be reported in the final trial protocol with reasons for drop out. Temporal deviations of up to 3 weeks, for example, in the case of illness of participants or SLPs, will not result in drop out and planned intervention or assessment sessions will be continued. At least $75 \%$ of the assigned homework must be completed until the last therapy session of each intervention period for participants to be included in the per protocol analysis. We will undertake all reasonable efforts for participant retention during the study period:

- Treatment locations close to participants' homes.

- Option to perform interventions at participants' homes.

- A worksheet for structuring homework.

- SLPs will support the participants in integrating the homework into their daily routines (eg, setting an alarm, asking family members to remind the participants, preparing memos) and call participants prior to follow up appointments, if requested.

\section{Data management}

A data management plan will be provided on Open Science Framework (OSF) ${ }^{53}$ on the publication of the final trial report. Subsequent to informed consent, the data described above will be recorded with an audiorecording device, transcribed and entered in SPSS. ${ }^{54}$ Collected data will be described by metadata and will be pseudonymised by assigning a number to each participant. The list that links personal data to other data will be destroyed for the sake of anonymisation after the data analysis. Data will be stored on a laptop, on an external hard-drive and on a decentral server of the university of Erfurt and will be archived for at least ten years on millenial discs. Backups will be implemented regularly.

\section{Statistical methods}

Primary and secondary analyses will be conducted using contrast analyses within repeated measures ANOVA models. We will apply a two-step approach to address the primary objective. First, changes in the primary outcome (TCT-D Total) will be analysed over time. Three contrasts (T2-T4; T2-T5; T2-T6) will be used to analyse whether the intervention led to any short-term and/or long-term benefits. Second, we will investigate superiority to spontaneous recovery by comparing the change scores from step one (T2-T4; T2-T5; T2-T6) with the change score from the waiting period (T1-T2). To address the secondary objectives, we will conduct the same analysis with the outcomes of the German version of CARA Reading Questionnaire to investigate superiority of the intervention with regard to reading activities. Furthermore, we will compare contrasts (T2- T3; T2-T4) between groups to explore strategy-specific effects on macrostructure and microstructure as well as on reading activities. Potential moderator effects will be explored using severity of aphasia, gender, age and education as moderators. We will use a 0.05 significance level for all models. We expect dropouts to be minimal. In case of missing data points, we will use listwise deletion and conduct analyses on just the 


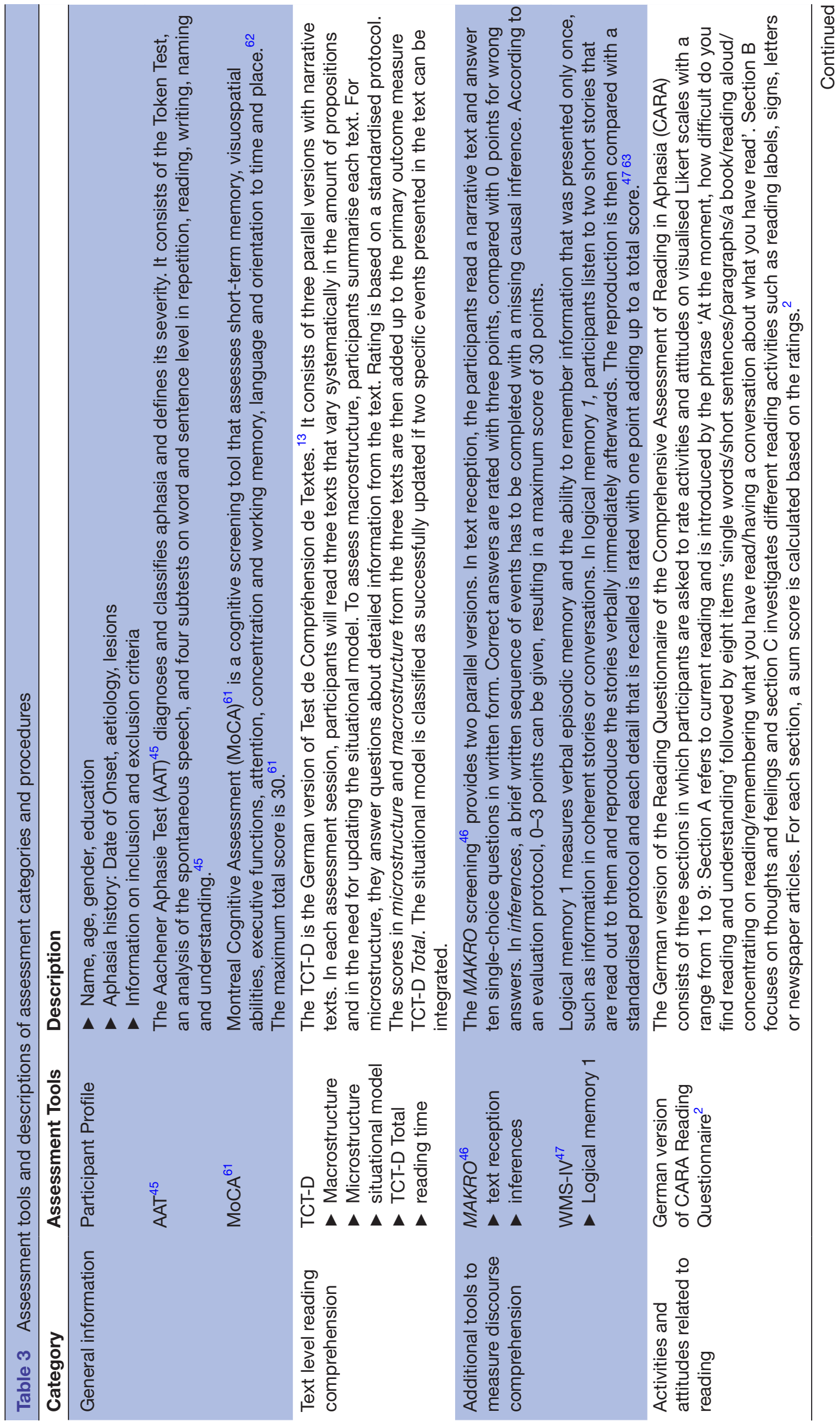




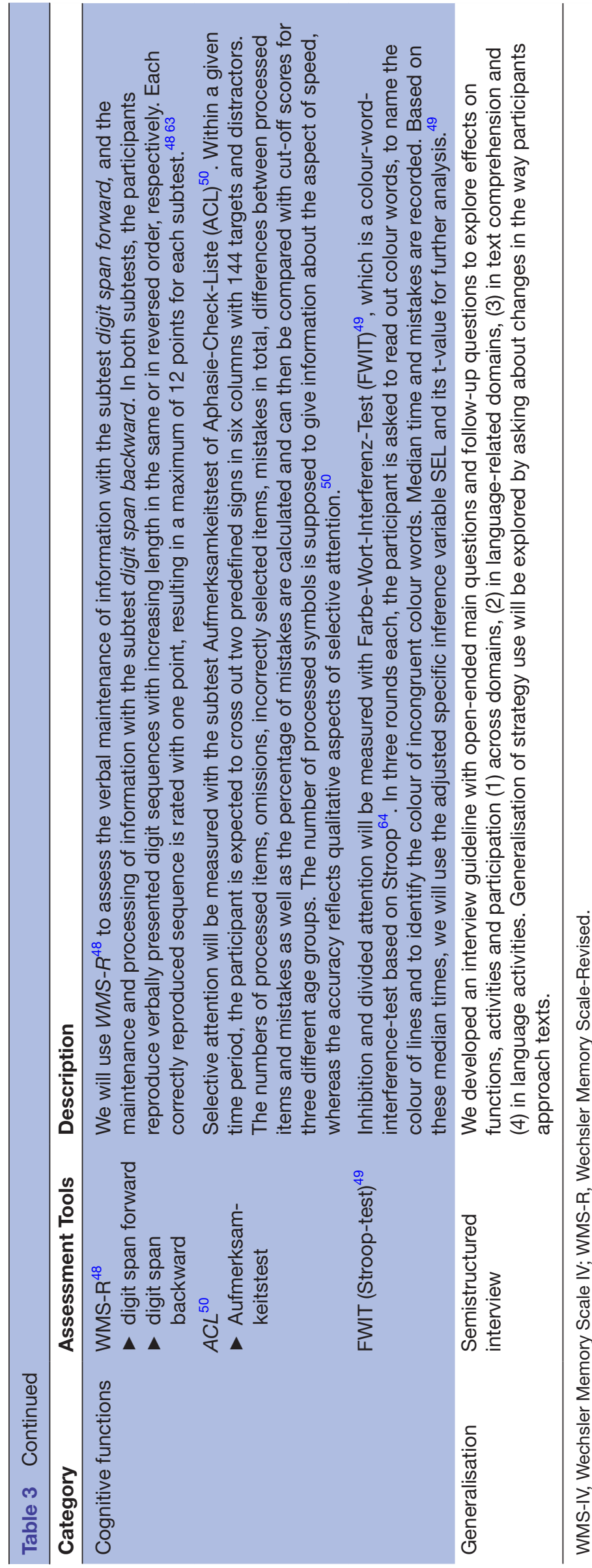




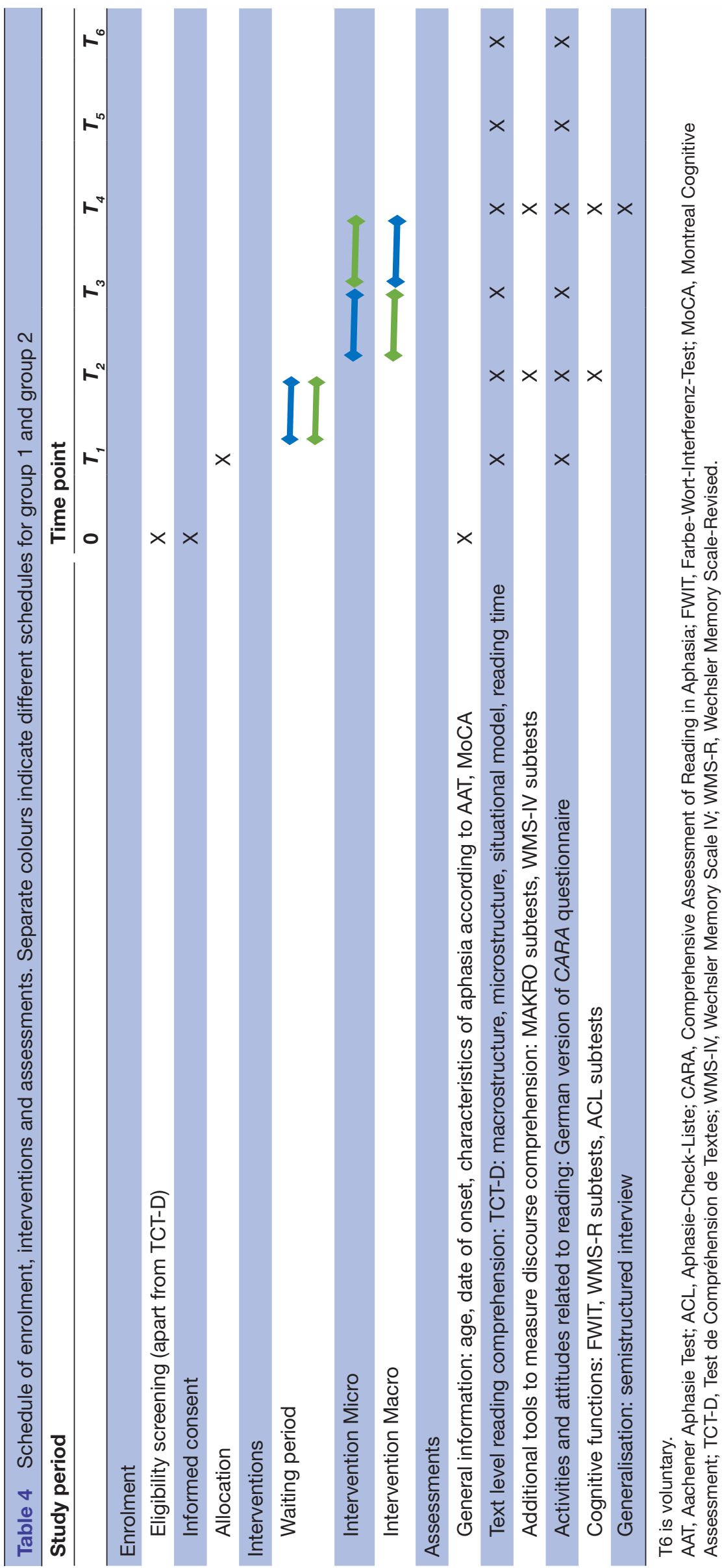

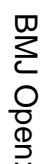

$\stackrel{\vec{P}}{\stackrel{9}{+}}$

등

$\frac{\bar{\sigma}}{\bar{D}}$

ळ

。

$\vec{\sigma}$

음.

8

กิ

京

N

옥

N

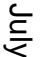

N

뭉

흥

$\frac{0}{8}$

혹

륭

홍.

$\frac{8}{9}$

혹.

옳

을.

N

N

ত্

$\stackrel{0}{\Phi}$

0

웅

잉 
metrics that have both pretreatment and post-treatment data points available.

\section{Monitoring}

ST or the Deutscher Bundesverband für akademische Sprachtherapie und Logopädie (dbs) ethics committee may withdraw particular participants if any new information indicates that continuing participation is not in their best interests. Adverse events or unintended effects reported by participants will be described in the final trial report. Adherence to the therapy manual will be monitored by ST through a standardised therapy documentation form and weekly supervision for participating SLPs. There will be neither independent auditing procedures, nor an official data monitoring committee or an interim analysis because the study is pure behavioural, noninvasive speech and language therapy and therefore, we expect no risks or harms.

\section{Patient and public involvement}

Patients and/or the public were not involved in the design, conduct, assessment of the burden, reporting or dissemination plans. Support groups with PWA will be involved in the recruitment of participants.

\section{Ethics and dissemination}

The study has been approved by the ethics committee of dbs (reference number: 20-10074-KA-MunmErw+Ko). Participants may be informed about their individual results after the final assessment on request. They will be advised on how to proceed in case of unexpected findings. There are no publication restrictions. Results will be disseminated as soon as possible regardless of the magnitude or the direction of effect in journals that focus on speech and language therapy or related subjects. Anonymised participant-level datasets and statistical codes will be provided on OSF repository after journal publication according to findable, accessible, interoperable and reusable principles with a digital object identifier and a CC-BY Creative Commons Licence. Authorship in the final trial report will be granted according to standards presented in McNutt $e t a \tilde{l}^{5}$. Professional writers and editorial services will not contribute to the study.

\section{Protocol amendments}

Important protocol modifications will be registered on Deutsches Register Klinischer Studien (see online supplemental table 1) and communicated to the ethics committee.

\section{Consent to participate}

SLPs will obtain informed consent from participants or their representatives with forms approved by the ethics committee (see online supplemental file 1 for English language example). Signed forms will be collected by ST.

\section{Consent for publication}

Not applicable. No details relating to individual persons have been included.

\section{Confidentiality}

Personal data will be collected on paper and on electronic data storage devices. Sensitive data will be stored in password-protected digital files or in a locked cabinet. Pseudonymised data may solely be shared according to specific regulations approved by the dbs ethics committee.

\section{DISCUSSION}

To date, evidence for text-level reading comprehension treatments in PWA remains rare and precludes sound clinical reasoning. In this study, we will investigate the effects of a strategy-based intervention on text level reading comprehension in a repeated measures study. The results will contribute to a better understanding of the role of cognitive functions and of reading strategies as well as to decision-making in text level reading comprehension treatments in PWA. Effects on specific reading functions and activities may allow SLPs to combine strategies according to individual reading profiles.

Due to the lack of a German-language tool to measure text level reading comprehension in PWA, we will use the translated version of the $\mathrm{TCT}^{13}$ for which no German normative data exists yet. We will address this issue by collecting data from a parallelised healthy reference group. Furthermore, we will use additional tools that have been validated and normalised with German samples in the assessment sessions $\mathrm{T} 2$ and $\mathrm{T} 4$ before and after the strategy-based intervention (see table 4).

Three parallel versions of the TCT-D exist, whereas at least five assessment sessions will take place. To estimate the possible impact of retest effects, each participant of the healthy parallelised reference group will be tested twice with the same version of the TCT-D in the same interval as participants of the intervention groups.

If we find positive effects of the strategy-based intervention, future research should compare strategy-based interventions with other treatment options in RCTs.

\section{Twitter Philipp Schmid @PhilippMSchmid}

Acknowledgements We would like to thank Dr. Thomas Lauer, scientific managing director of ErfurtLab, who provided his expertise on randomisation processes and who will perform the randomisation and allocation procedures. Thanks to Prof. Dr. Andreas Mayer, Ludwig-Maximilians-University Munich, and to Stiftung Pfennigparade, Therapiepraxis, for providing access to facilities for speech and language therapy. Furthermore, we express our gratitude to Janet Webster and her colleagues who supported the process of translation and adaptation of the Reading Questionnaire of CARA [2], to Niamh Petri who supported its back translation, to Antonia Wolf for feedback from an SLP's perspective on its German version as well as to Corinna Thomas, Nora Bauer and Rebecca Neueder for supporting testruns with its pilot version. Thanks also to Luisa Scherf and Jula Carlsen for their valuable comments on the therapy manual and to Rahel Wacker, who prepared two beautiful drawings for the therapy manual. Thanks to Jan Stratil for helpful discussions about the study design, to Maria Ewald and Anna Thomas for performing pretests with TCT-D, and to Sofie Bale for discussions about wording and orthography in British English. Thank you to the peer reviewers for their helpful and constructive comments.

Contributors S-MT conceived the study and led the protocol development. S-MT, PS, SC and FD developed the study design. FD helped with implementation. SC supported the translation and adaptation process of TCT (13). S-MT is responsible for data acquisition and data management. PS provided statistical expertise in clinical trial design. All authors contributed to refinement of the study protocol and 
approved the final manuscript. No other groups or individuals will be involved in overseeing the trial.

Funding This work was supported by a Christoph-Martin-Wieland scholarship of the University of Erfurt granted to S-MT.

Disclaimer This source of funding did not and will not influence any aspects in study design, conduct, data analysis, interpretation, manuscript writing, and dissemination of results.

Competing interests None declared.

Patient consent for publication Not required.

Provenance and peer review Not commissioned; externally peer reviewed.

Supplemental material This content has been supplied by the author(s). It has not been vetted by BMJ Publishing Group Limited (BMJ) and may not have been peer-reviewed. Any opinions or recommendations discussed are solely those of the author(s) and are not endorsed by BMJ. BMJ disclaims all liability and responsibility arising from any reliance placed on the content. Where the content includes any translated material, BMJ does not warrant the accuracy and reliability of the translations (including but not limited to local regulations, clinical guidelines, terminology, drug names and drug dosages), and is not responsible for any error and/or omissions arising from translation and adaptation or otherwise.

Open access This is an open access article distributed in accordance with the Creative Commons Attribution Non Commercial (CC BY-NC 4.0) license, which permits others to distribute, remix, adapt, build upon this work non-commercially, and license their derivative works on different terms, provided the original work is properly cited, appropriate credit is given, any changes made indicated, and the use is non-commercial. See: http://creativecommons.org/licenses/by-nc/4.0/.

\section{ORCID iD}

Frank Domahs http://orcid.org/0000-0002-5583-4681

\section{REFERENCES}

1 Brookshire CE, Wilson JP, Nadeau SE, et al. Frequency, nature, and predictors of alexia in a convenience sample of individuals with chronic aphasia. Aphasiology 2014;28:1464-80.

2 Webster J, Morris J, Malone J, et al. Reading comprehension difficulties in people with aphasia: investigating personal perception of reading ability, practice, and difficulties. Aphasiology 2021;35:805-23.

3 Simmons-Mackie N, Kagan A. Application of the ICF in aphasia. Semin Speech Lang 2007;28:244-53.

4 Webster J, Samouelle A, Morris J. The brain can't cope': Insights about reading from people with chronic aphasia. Available: https:// doi.org/10.31234/osf.io/p8xh4

5 World Health Organisation, ICF. Internationale Klassifikation der Funktionsfähigkeit, Behinderung und Gesundheit, Stand Oktober 2005. Deutsches Institut für Medizinische Dokumentation und Information, 2005. Available: https://www.dimdi.de/dynamic/. downloads/klassifikationen/icf/icfbp2005.zip

6 McKevitt C, Fudge N, Redfern J, et al. Self-Reported long-term needs after stroke. Stroke 2011;42:1398-403.

7 Purdy M, Coppens P, Madden EB, et al. Reading comprehension treatment in aphasia: a systematic review. Aphasiology 2019;33:629-51.

8 Watter K, Copley A, Finch E. Discourse level reading comprehension interventions following acquired brain injury: a systematic review. Disabil Rehabil 2017;39:315-37.

9 Jesch T. Textverstehen. In: Garbe C, Holle K, Jesch T, eds. Texte lesen: Textverstehen - Lesedidaktik - Lesesozialisation. Paderborn: Ferdinand Schöningh, 2010: 39-102.

10 Rosebrock C, Nix D. Grundlagen der Lesedidaktik und der systematischen schulischen Leseförderung. 4th ED. Baltmannsweiler: Schneider, 2011.

11 Perfetti C. Comprehending written language: a blueprint of the reader. In: Brown CM, Hagoort P, eds. The Neurocognition of language. Oxford, New York: Oxford University Press, 1999: 167-208.

12 Kintsch W. Revisiting the construction-integration model of text comprehension and its Implications for Instruction. In: Alvermann DE, Unrau NJ, Sailors M, et al, eds. Theoretical models and processes of literacy. New York: Routledge, 2018: 178-203.

13 Chesneau S. TCT, Test de Compréhension de Textes: 16-80 ans. Paris: Mot à Mot, 2012.
14 Guthke T. Psychologische Untersuchungen zu Inferenzen beim Satzund Textverstehen [dissertation] 1992.

15 Chesneau S, Ska B. Text comprehension in residual aphasia after basic-level linguistic recovery: a multiple case study. Aphasiology 2015;29:237-56.

16 Meteyard L, Bruce C, Edmundson A, et al. Profiling text comprehension impairments in aphasia. Aphasiology 2015;29:1-28.

17 Ziegler W. Leitlinien für Diagnostik und Therapie in der Neurologie. Rehabilitation aphasischer Störungen nach Schlaganfall, 2015. Available: https://www.dgn.org/images/red_leitlinien/LL_2012/pdf/ 030-090I_S1_Schlaganfall_Rehabilitation_aphasischer_St\%C3\% B6rungen 2013 verlaengert.pdf

18 Klingenberg G. Das Verarbeiten von Texten bei Aphasie: Untersuchungen zur modalitätsspezifischen Verarbeitung narrativer Texte. Hochschulverlag: Freiburg, 1997.

19 Riedel B. Texte für die neurologische Rehabilitation. Hofheim: NAT, 2014.

20 Cherney LR. Oral reading for language in aphasia: impact of aphasia severity on cross-modal outcomes in chronic nonfluent aphasia. Semin Speech Lang 2010;31:042-51.

21 Katz RC, Wertz RT. Computerized hierarchical reading treatment in aphasia. Aphasiology 1992;6:165-77.

22 Katz RC, Wertz RT. The efficacy of computer-provided reading treatment for chronic aphasic adults. J Speech Lang Hear Res 1997;40:493-507.

23 Coelho C. Direct attention training as a treatment for reading impairment in mild aphasia. Aphasiology 2005;19:275-83.

24 Lee JB, Moore Sohlberg M, Sohlberg MM. Evaluation of attention training and metacognitive facilitation to improve reading comprehension in aphasia. Am J Speech Lang Pathol 2013;22:S318-33.

25 Sinotte MP, Coelho CA. Attention training for reading impairment in mild aphasia: a follow-up study. NeuroRehabilitation 2007;22:303-10.

26 Mayer JF, Murray LL. Approaches to the treatment of alexia in chronic aphasia. Aphasiology 2002;16:727-43.

27 Artelt C, McElvany N, Christmann U. Expertise - Förderung von Lesekompetenz. Bildungsreform 2005;17.

28 Mayer A, Marks D-K. Förderung des Textverständnisses durch die Vermittlung von Verstehensstrategien - Eine Metaanalyse zur Effektivität. Improving reading comprehension skills by mediating comprehension strategies. A meta-analysis of the effectiveness. Forschung Sprache 2019;7:4-36.

29 Souvignier E, Antoniou F. Förderung des Leseverständnisses bei Schülerinnen und Schülern mit Lernschwierigkeiten - eine Metaanalyse. Vierteljahreszeitschrift für Heilpädagogik und ihre Nachbargebiete 2007;76:46-62.

30 Lynch KE, Damico JS, Abendroth KJ, et al. Reading performance subsequent to aphasia: strategies applied during authentic reading Aphasiology 2013;27:723-39.

31 Cocks N, Pritchard M, Cornish $\mathrm{H}$, et al. A "novel" reading therapy programme for reading difficulties after a subarachnoid haemorrhage. Aphasiology 2013;27:509-31.

32 Gold PC, Freeman EA. Remediation of alexia: a case study. Read Psychol 1984;5:65-73.

33 Lynch KE, Damico JS, Damico HL, et al. Reading skills in an individual with aphasia: the usefulness of Meaning-Based clinical applications. Asia Pac J Speech Lang Hear 2009;12:221-34.

34 Webster J, Morris J, Connor C, et al. Text level reading comprehension in aphasia: what do we know about therapy and what do we need to know? Aphasiology 2013;27:1362-80.

35 Rogalski Y, Edmonds LA. Attentive reading and constrained Summarisation (ARCs) treatment in primary progressive aphasia: a case study. Aphasiology 2008;22:763-75.

36 Rogalski Y, Edmonds LA, Daly VR, et al. Attentive reading and constrained Summarisation (ARCS) discourse treatment for chronic Wernicke's aphasia. Aphasiology 2013;27:1232-51.

37 Sohlberg MM, Griffiths GG, Fickas S. An evaluation of reading comprehension of expository text in adults with traumatic brain injury. Am J Speech Lang Pathol 2014;23:160-75.

38 Schmidt C. Lesestrategien. Französisch heute 2007;38:121-9.

39 Gudjons H. Handlungsorientiert lehren und lernen: Schüleraktivierung - Selbsttätigkeit - Projektarbeit. 8th ed. Bad Heilbrunn: Klinkhardt, 2014.

40 Chan A-W, Tetzlaff JM, Altman DG, et al. Spirit 2013 statement: defining standard protocol items for clinical trials. Ann Intern Med 2013:158:200-7.

41 Faul F, Erdfelder E, Buchner A, et al. Statistical power analyses using $\mathrm{G}^{*}$ Power 3.1: tests for correlation and regression analyses. Behav Res Methods 2009;41:1149-60.

42 Breitenstein C, Grewe T, Flöel A, et al. Intensive speech and language therapy in patients with chronic aphasia after stroke: a randomised, 
open-label, blinded-endpoint, controlled trial in a health-care setting. Lancet 2017;389:1528-38.

43 Connor LT, Obler LK, Tocco M, et al. Effect of socioeconomic status on aphasia severity and recovery. Brain Lang 2001;78:254-7.

44 Nakagawa Y, Sano Y, Funayama M, et al. Prognostic factors for long-term improvement from stroke-related aphasia with adequate linguistic rehabilitation. Neurol Sci 2019;40:2141-6.

45 Huber W, Poeck K, Weniger D. Aachener Aphasie Test (AAT). Göttingen: Hogrefe, 1983.

46 Büttner J. MAKRO: Screening zur Verarbeitung der Makrostruktur von Texten bei neurologischen Patienten. Hofheim: NAT-Verlag, 2018.

47 Petermann F, Lepach AC. Wechsler memory scale (WMS-IV): Manual zur Durchführung und Auswertung; deutsche Übersetzung und Adaptation der WMS-IV von David Wechsler. 4 ed. Frankfurt/M: Pearson, 2012.

48 Härting C, Markowitsch HJ, Neufeld H. Wechsler-Gedächtnistest - revidierte Fassung: WMS-R; Manual; deutsche Adaptation der revidierten Fassung der Wechsler Memory scale. 1st ed. Bern: Huber, 2000.

49 Bäumler G. FWIT Farbe-Wort-Interferenz-Test nach J. R. Stroop. Göttingen: Hogrefe, 1985.

50 Kalbe E, Reinhold N, Ender U. Aphasie-Check-Liste (ACL). Köln: ProLog, 2002.

51 Boutron I, Guittet L, Estellat C, et al. Reporting methods of blinding in randomized trials assessing nonpharmacological treatments. PLOS Med 2007;4:e61.

52 Karanicolas PJ, Farrokhyar F, Bhandari M. Practical tips for surgical research: blinding: who, what, when, why, how? Can J Surg 2010;53:345-8.
53 Center for Open Science. OSF, 2020. Available: https://osf.io/ 54 IBM Corp. IBM SPSS Statistics for Windows. 27. Armonk, NY: IBM Corp., 2019.

55 McNutt MK, Bradford M, Drazen JM, et al. Transparency in authors contributions and responsibilities to promote integrity in scientific publication. Proc Natl Acad Sci U S A 2018;115:2557-60.

56 Nguyen $\mathrm{H}$, Morris $\mathrm{J}$, Webster $\mathrm{J}$, et al. Reading of everyday texts by people with aphasia: do advance organisers help? Aphasiology 2018;32:153-5.

57 Koppensteiner J. Literatur im DaF-Unterricht: eine Einführung in produktiv-kreative Techniken. 1st ed. Wien: öbv et hpt, 2001.

58 Frieg H, Hilbert C, Belke E. Sprachförderung in ein- und mehrsprachigen Gruppen: die generative Textproduktion. Sprachheilarbeit 2012;57:155-61.

59 Belke G. Mehrsprachigkeit im Deutschunterricht: Sprachspiele, Spracherwerb und Sprachvermittlung. 4th ed. Baltmannsweiler: Schneider Verlag Hohengehren, 2008.

60 Höllein D. Valency theory. in: linguistics. Oxford: Oxford University Press, 2020. https://www.oxfordbibliographies.com/view/document/ obo-9780199772810/obo-9780199772810-0260.xml

61 Bartusch S, Zipper S. Montreal cognitive assessment (MoCA) Deutsche Übersetzung, 2004. Available: www.mocatest.org

62 Nasreddine Z. Moca Montreal cognitive assessment. MoCA Montreal cognitive assessment, 2019. Available: https://www.mocatest.org/ the-moca-test/

63 Schellig P, Drechsler R, Heinemann D, et al, eds. Aufmerksamkeit, Gedächtnis, exekutive Funktionen. 1st ed. Göttingen: Hogrefe, 2009.

64 Stroop JR. Studies of interference in serial verbal reactions. J Exp Psychol 1935;18:643-62. 